homeostasis, reactive oxygen species production and, to a lesser extent, ATP generation. The balance of mitochondrial fission, fusion and motility is likely to provide fine control of subcellular location and interactions of the organelle; however, the outcome of perturbation of mitochondrial dynamics on endothelial function remains unclear. We sought to address this gap by investigating the effects of mitochondrial fission inhibitor, Mdivi-1, on endothelial cells.

Treatment of cultured endothelial cells with Mdivi-1 (1 or $10 \mu \mathrm{M}, 48$ hour) increased mitochondrial length and branching extent compared to control, consistent with inhibition of fission. Mdivi-1 increased branched, twisted and looped endothelial mitochondrial morphologies, whilst also reducing net mitochondrial speed. No acute toxicity was observed after Mdivi-1 treatment $(10 \mu \mathrm{M}, 48$ hour), however Mdivi-1 did decrease the intracellular content of the glycoprotein von Willebrand Factor (produced, stored and released by endothelial cells to aid thrombosis). Endothelial gap junction communication was also assessed as a function of confluent cells' ability to inter-cellularly transfer the dye, Lucifer yellow; Mdivi-1 decreased dye transfer rates, suggesting reduced intercellular gap junction communication.

In conclusion, Mdivi-1 treatment altered endothelial mitochondrial morphology and dynamics, and also decreased von Willebrand Factor content and gap junction communication, however the mechanistic links remain unclear. Clarification is important, as modulation of mitochondrial dynamics has been proposed as a novel target against the cell proliferation associated with vascular disease.

\section{P27 DEPLETION OF CARDIAC SUCCINATE MEDIATES IMPAIRED HYPOXIA-INDUCIBLE FACTOR 1A SIGNALLING BY LONG CHAIN FATTY ACIDS IN INSULIN RESISTANCE}

${ }^{1} \mathrm{MS}$ Dodd, ${ }^{1} \mathrm{Mdl}$ Sousa Fialho, ${ }^{1} \mathrm{CN}$ Montes Aparico, ${ }^{1} \mathrm{M}$ Kerr, ${ }^{1} \mathrm{KN}$ Timm, ${ }^{2} \mathrm{H}$ Griffin, ${ }^{3}$ JJFP Luiken, ${ }^{3}$ JFC Glatz, ${ }^{1} \mathrm{DJ}$ Tyler, ${ }^{1} \mathrm{LC}$ Heather. 'Department of Physiology, Anatomy and Genetics, University of Oxford, Parks Road, Oxford, UK; ${ }^{2}$ Department of Biochemistry, University of Cambridge, Cambridge, UK; ${ }^{3}$ Department of Molecular Genetics, Cardiovascular Research Institute Maastricht (CARIM), Maastricht University, Maastricht, The Netherlands

10.1136/heartjnl-2018-BSCR.32

Hypoxia-inducible factor (HIF) $1 \alpha$ is activated following myocardial infarction, and is critical for cell survival in hypoxia. In cancer, changes in Krebs cycle intermediates have also been shown to affect HIF1 $\alpha$ stabilisation. We questioned whether abnormal metabolism could prevent HIF $1 \alpha$ activation in diabetes. Type 2 diabetic hearts have decreased HIF $1 \alpha$ protein accumulation in ischemia, which correlated negatively with plasma fatty acid (FA) concentrations and positively with myocardial succinate concentrations. In insulin-resistant cardiomyocytes, HIF signalling and downstream metabolic adaptation was suppressed in hypoxia. Impaired HIF $1 \alpha$ stabilisation was due to increased degradation of the protein in hypoxia, as inhibition of the proteasome or inhibition of the HIF hydroxylases was able to increase HIF1 $\alpha$ in insulin resistance. This was due to abnormal metabolism, as FA (both palmitate and oleate) prevented HIF1 $\alpha$ accumulation in a concentration-dependent manner, which could be reversed by blocking CD36 mediated FA uptake. Succinate promotes HIF stabilisation by inhibiting the HIF hydroxylases, however, FA suppressed succinate accumulation during hypoxia. Increasing succinate concentrations using dimethylfumarate, overrides the FA-mediated inhibition of HIF $1 \alpha$ in a concentration-dependent manner. Pharmacologically inhibiting the HIF hydroxylases promoted HIF $1 \alpha$ accumulation and improved cardiac function following ischemia-reperfusion in diabetic rats. In conclusion, elevated FA in type 2 diabetes prevent HIF $1 \alpha$ accumulation by decreasing succinate concentrations in hypoxia.

\section{P28 IDENTIFICATION OF A SHEAR STRESS RESPONSIVE NOVEL GENE IN THE INTRON OF LAF4}

M Ehteramyan, L Yi, Y Hu, L Zeng. Cardiovascular division, James Black Centre, King's College London, London, UK

\subsection{6/heartjnl-2018-BSCR.33}

A novel gene was recently discovered using microarray gene profiling of shear stress-induced genes in mouse embryonic stem cells (ESCs), which is located within the intron of the transcription factor Laf4 and therefore designated as Laf4 intron resident gene (Laf4ir). Laf4 and Laf4ir genes use different strands for transcription. Two transcript variants have been identified for Laf4ir with three potential opening reading frames (ORFs). In this study, we intend to characterise the transcriptional/translational expression, regulation, and functions of this novel gene. The experiments with qRT-PCR analysis revealed that Laf4ir mRNA was unregulated transiently by shear stress compared to static conditions in mouse differentiated ESCs. Laf4ir was expressed in late stage of mouse embryos but not in the early stages and Laf4ir was differentially expressed in adult mice organs/tissues. Specific antibodies against peptides from the ORFs confirmed the translation of these ORFs in mouse differentiated ESCs in response to shear stress. Immunofluorescence staining with antibody against ORF2 revealed that the ORF2 was exclusively expressed in the intima of aorta in wild type mice. Over-expression of ORF2 significantly increased CD31 expression in mouse ESC-derived Sca1+ cells, indicating the potential role of Laf4ir in endothelial cell differentiation. Interestingly, high level of ORF2 was detected from intima to the adventitia of aorta in ApoE-/- mice and in the adventitia of femoral artery from platinum wire injured mice. Further detailed and concrete investigation is required to characterise the potential functions of Laf4ir gene products, which may provide some new insights into vascular biology.

\section{P29 THE TRANSCRIPTION FACTOR BRN-3B/(POU4F2) REGULATES VASCULAR FUNCTION AND INTEGRITY IN VIVO}

L Mele, $\amalg$ Maskell, VS Budhram-Mahadeo. Medical Molecular Biology Unit, Institute of Cardiovascular Science, University College London, UCL, Rayne Building, London, UK

\subsection{6/heartjnl-2018-BSCR.34}

Rationale Chronic hypertension is a major risk factor for stroke and coronary heart disease. Hypertension is associated with vascular smooth muscle cell (VCMCs) dysfunction, leading to vascular wall calcification and reduced vessel compliance. However, the underlying cellular mechanisms remain unclear. We previously demonstrated that loss of the transcription factor Brn-3b/(POU4F2) results in weight gain and diabetes in mice, known risk factors for hypertension. Herein we present evidences for a direct role of Brn-3b in the maintenance of vascular integrity. 